\title{
Measurements of Coastal Deviation of High-Frequency Radio Waves
}

\author{
C. W. McLeish ${ }^{1}$
}

(August 4, 1959)

\begin{abstract}
The angular deviation of the phase front of a wave propagated across a fresh water shoreline has been measured over the frequency range from 3 to $20 \mathrm{Mc}$. The deviation is found to be roughly half that which theoretically would be obtained if the same sites were adjacent to infinitely conducting surfaces.
\end{abstract}

\section{Introduction}

Some recent measurements of coastal deviation at low frequency (lf) have been reported by Pressey, Ashwell, and Fowler $[1,2]^{2}$. Their results indicate the practical difficulty of interpreting measured phase front deviations. Irregular coastline and nonhomogeneous soil constants create wave interference effects which tend to mask the deviation due to the boundary.

This paper presents measurements made at high frequency (hf) (3 to $20 \mathrm{Mc}$ ) where the site area which affects the results can be much smaller than in the lf case. Observations were made over a number of paths at each site, thus reducing to some extent the site error.

Finally, a comparison is drawn between the experimental and the theoretical deviations which would be expected under certain ideal conditions.

\section{Observations}

Two sites were chosen, each with a reasonably straight shoreline along a river but with quite different soil constants. Measured values are given in table 1 for both sites and for the river water.

TABLE 1. Site ground constants

\begin{tabular}{|c|c|c|c|}
\hline Site & Method & $\begin{array}{l}\text { Conduc- } \\
\text { tivity, } \\
\sigma \text { emu }\end{array}$ & $\begin{array}{c}\text { Relative } \\
\text { permit- } \\
\text { tivity, } K\end{array}$ \\
\hline $\begin{array}{l}1 \text { arable and } \\
2 \text { \{limestone-...- } \\
\text { River water.... }\end{array}$ & $\begin{array}{l}\text { Wave tilt } \\
\text { Wave tilt } \\
\text { Surface sample } \\
\text { Sample }\end{array}$ & $\begin{array}{l}1 \times 10^{-13} \\
3 \times 10^{-14} \\
3 \times 10^{-16} \\
1 \times 10^{-13}\end{array}$ & $\begin{array}{r}9 \\
25 \\
7 \\
81\end{array}$ \\
\hline
\end{tabular}

The wave tilt measurements at site 2 are of doubtful accuracy because the path of propagation was within a half wavelength of the water at the low frequencies where a value could be obtained. However, it is likely that both conductivity and relative permittivity will be higher than that obtained in samples because of water in fissures in the rock.

\footnotetext{
1 Division of Electrical and Radio Engineering, National Research Council, Ottawa, Canada.

${ }_{2}^{2}$ Figures in brackets indicate the literature references at the end of this paper.
}

A coaxial-spaced loop direction finder was used to measure the deviations of the phase front of the horizontal magnetic field. The transmitter in all cases was situated over the water. In order to eliminate systematic instrumental error, the difference between a radio bearing and the visual bearing was observed first on a path normal to the shoreline and then on a path usually at $60^{\circ}$ to the normal. Observations were made at $1-\mathrm{Mc}$ intervals in the 3to $20-\mathrm{Mc}$ band.

At site 1 readings were taken from eight positions, all approximately $40 \mathrm{~m}$ from the shoreline. The mean of the deviations is plotted in figure 1(a). The

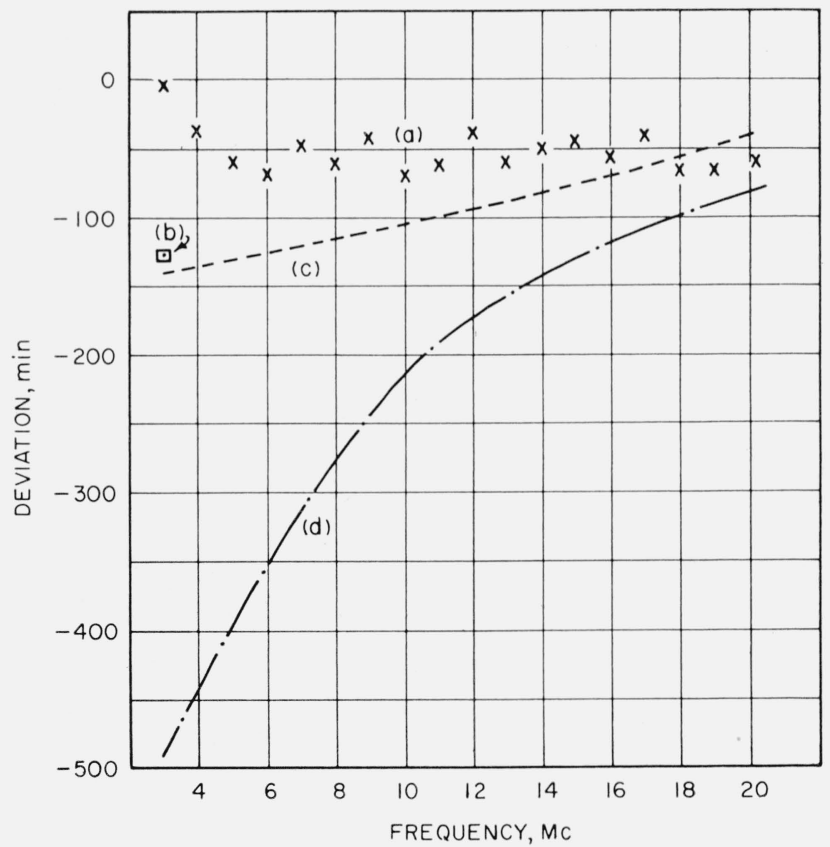

Figure 1. Coastal deviations at site 1 for $\theta=60^{\circ}$.

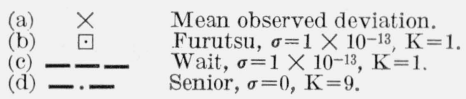

rms fluctuation of individual deviations about the mean is about $43 \mathrm{~min}$, indicating the effects of site error on the result. 
At site 2, five sets of readings were obtained from positions $30 \mathrm{~m}$ from the shoreline and two sets at $10 \mathrm{~m}$. The mean deviations are plotted in figure 2 (a) and (f). The site error contribution appeared to be about the same as at site 1 .

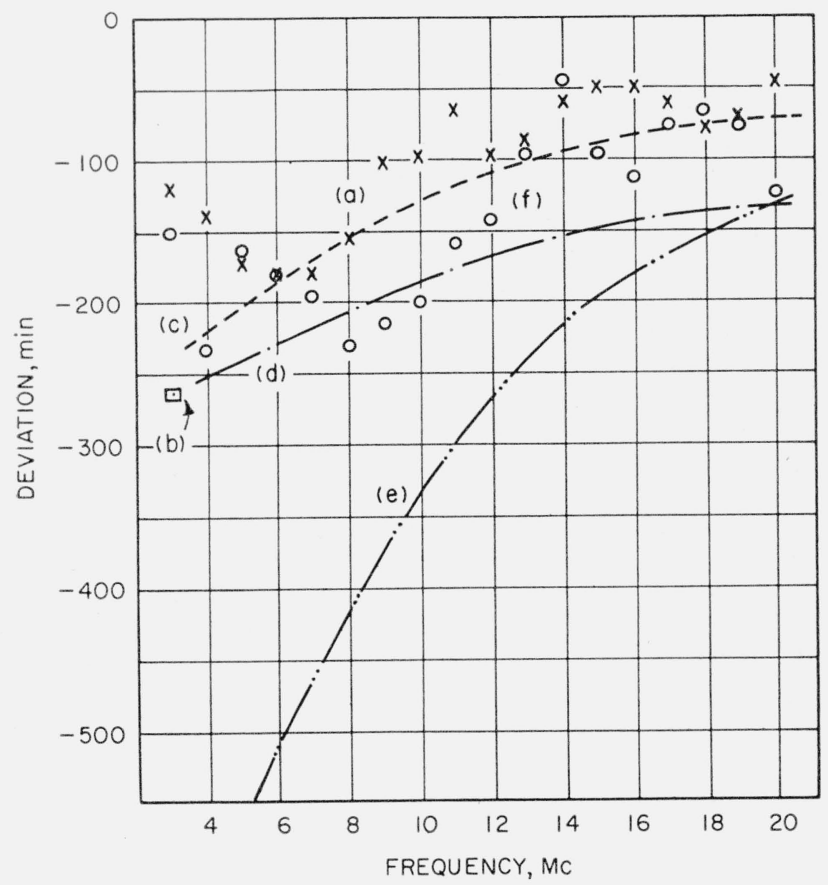

Figure 2. Coastal deviations at site 2 for $\theta=60^{\circ}$.

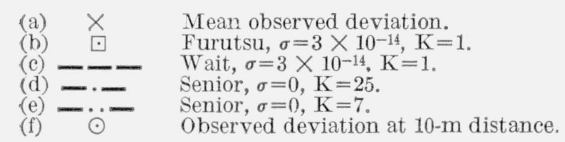

\section{Theory}

Several papers [3 to 8] deal with the refraction of a surface wave crossing the boundary between two media. The general expressions for the angular deviation of the wave front are not capable of numerical solution and in each case simplifying assumptions have to be made to arrive at values for the deviation. For instance, Furutsu [6] provides an expression for deviation when one medium is infinitely conducting, the other has finite conductivity, and the wave path numerical distance of the receiver from the boundary is small. Using parametric curves, Wait [7] has derived an expression allowing for finite conductivity on both sides of the boundary and for unrestricted distances. Senior [8] has investigated the deviation when one medium is infinitely conducting and the other a lossless dielectric. Whereas the first two cases apply chiefly to the low frequencies for groundwave propagation, the last one is applicable on higher frequencies. All the solutions require the distance from the boundary to be sufficiently large that only the radiation field is effective. Table 2 shows the expressions developed in the above papers, together with the limits imposed on each.

Apart from the dimensions defined in figure 3, where distances are in meters, the symbols used are defined as follows:

$f=$ frequency, cps,

$\lambda=$ wavelength, meters,

$K=$ relative permittivity,

$\rho_{2}=$ numerical distance along $r_{2},=\frac{\pi r_{2}}{x} \cos b$ for vertical polarization,

$x=\frac{1.8 \times 10^{21} \sigma}{f}$,

$b=\arctan \frac{K+1}{x}$

$\sigma=$ conductivity, emu,

$V=\frac{r_{2}}{r_{12}}$

$\delta V=\frac{-d \phi}{d V}$, obtained from Wait's curves of phase versus numerical distance, and

$\gamma=\frac{2 \pi}{\lambda}$ and $\eta=\frac{1}{\sqrt{K}}$ in this case.

TABLE 2. Theoretical deviations

\begin{tabular}{|c|c|c|c|c|c|}
\hline \multirow[b]{2}{*}{ Author } & Deviation angle & \multicolumn{2}{|c|}{ Conductivity } & \multicolumn{2}{|c|}{ Distance } \\
\hline & $\delta_{2}$, Degrees & 1st Medium & 2d Medium & $\begin{array}{l}\text { Numerical } \\
\rho_{2}\end{array}$ & $\begin{array}{l}\text { Normal } \\
\frac{2 \pi r_{2} \cos \theta}{\lambda}\end{array}$ \\
\hline Furutsu.... & $\frac{3.73 \tan \theta \sqrt{r_{1} / r_{2}}}{\sqrt{\sigma r_{12}}}$ & $\begin{array}{c}e m u \\
\infty\end{array}$ & $>>\frac{f K}{1.8 \times 10^{21}}$ & $<<1$ & $>>1$ \\
\hline Wait_.... & $\frac{\tan \theta(\delta V)(1-V)}{2 \pi r_{12}} \cdot \frac{180}{\pi}$ & $>>\frac{f K}{1.8 \times 10^{21}}$ & $>>\frac{f K}{1.8 \times 10^{21}}$ & (s) & $>>1$ \\
\hline Senior (eq 26) .. & $\arctan \frac{-\tan \ddot{\theta}}{4 r_{2}\left(\eta \sqrt{\frac{\gamma r_{2}}{\pi}}+\frac{1}{2 \eta} \sqrt{\frac{\pi}{\gamma r_{2}}}-1\right)}$ & $\infty$ & $<<\frac{f K}{1.8 \times 10^{21}}$ & n & $>>1$ \\
\hline
\end{tabular}

a Curves are given up to $\rho_{2}=5.0$. 


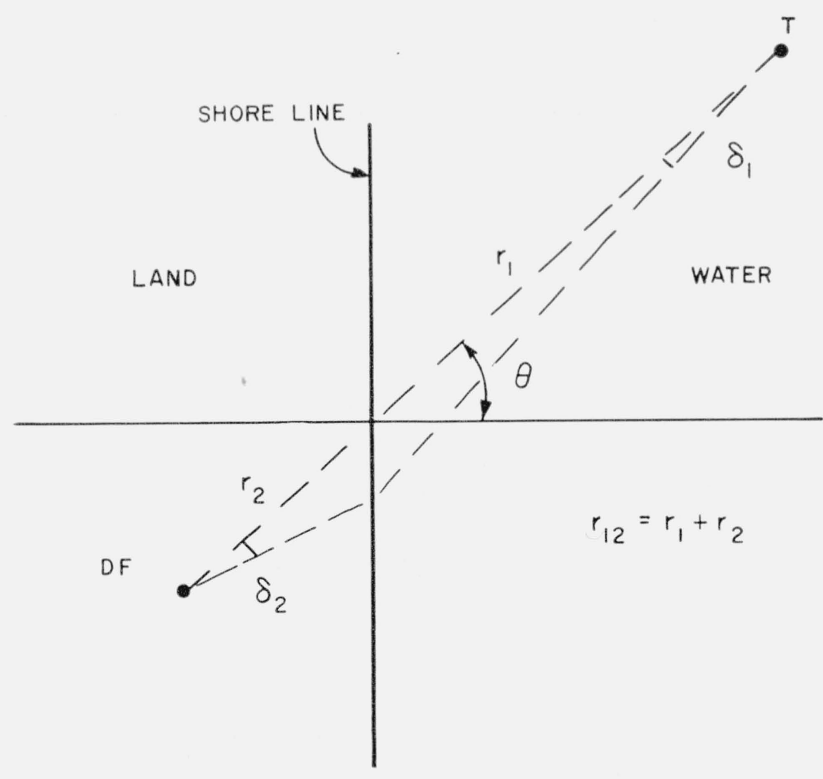

Figure 3. Geometry of deviation effect.

Senior's eq (26) refers to the vertical electric field rather than to the horizontal magnetic field deviations which are measured in the experiment.

For purposes of comparison, calculations of deviation for the three expressions have been carried out assuming the water path to be a perfect conductor in each case. Actually the water behaves as a dielectric medium above $2.2 \mathrm{Mc}$ because of its low conductivity. The deviation, according to Furutsu, is plotted for both sites in figures 1 (b) and 2(b) for $3 \mathrm{Mc}$ only, for at higher frequencies the numerical path $\rho_{2}$ is no longer small. In the same figures the curves (c) and (d) are for Wait's and Senior's expressions, respectively, using the indicated values of conductivity and permittivity. Curve (e) in figure 2 is for the measured sample value of relative permittivity.

\section{Discussion of Results}

At site 1 there is sufficiently high conductivity that the propagation constant does not become dependent on permittivity until the frequency is above $20 \mathrm{Mc}$. Therefore, the observed deviations should approach those given by Wait, except near the low end of the band where the water propagation constant is changing rapidly to that of a lossy medium having the same conductivity as the site. This may be the reason for the observed reduction of deviation at $3 \mathrm{Mc}$.

At site 2 propagation over the whole frequency range is affected chiefly by its permittivity. Therefore, one would expect deviations approaching those given by Senior. The observed deviations have a similar trend above $6 \mathrm{Mc}$ but are considerably smaller than expected for the ideal case. The difference may be partly because the measurements refer to the horizontal magnetic field while the theoretical curve refers to the vertical electric field, and partly be- cause the water permittivity is finite. The drop of deviation below $6 \mathrm{Mc}$ may again be attributed to the change of propagation over the water at low frequencies.

The same general trend is also apparent in figure 2 (f) and the deviations are somewhat larger. The distance from the shoreline in this case is, of course, too small to permit comparison with any of the theoretical expressions.

\section{Conclusions}

In the siting of direction finding stations and similar navigational aids near bodies of water, deviations of the wave front may introduce error in observations. All three theoretical expressions show that the angular deviation of the wave is approximately proportional to $\tan \theta$ and inversely proportional to $r_{2}$. Therefore, at large values of $\dot{\theta}$, where the error may be considered significant, it is approximately inversely proportional to $r_{2} \cos \theta$, the normal distance of the receiver from the boundary. By extrapolation from the observed results one might expect deviation errors on a high conductivity site adjacent to fresh water to be less than $1 / 2$ deg if the distance from the shoreline were at least $80 \mathrm{~m}$. Similarly on a site of very low conductivity, where the observed errors had a definite frequency dependence, the required distance for the same error limit is about 4 wavelengths. By comparison, sites beside the sea would, according to the theory, have to be about twice as far from the shoreline.

The author thanks J. McDougall and J. Lee for carrying out the experimental work.

\section{References}

[1] B. G. Pressey, G. E. Ashwell, C. S. Fowler, Change of phase with distance of a low frequency ground wave propagated across a coast line, Proc. Inst. Elec. Engrs., Paper 2082 R 103, Pt. B, 527 (July 1956).

[2] B. G. Pressey, G. E. Ashwell, The deviation of low frequency ground waves at a coastline, Proc. Inst. Elec. Engrs., Paper 2083 R 103, Pt. B, 535 (July 1956).

[3] P. C. Clemmow, Radio propagation over a flat earth across a boundary separating two different media, Phil. Trans. Roy. Soc. London, Ser. A 246, 1 (June 1953).

[4] G. Grunberg, Suggestion for a theory of coastline refraction, Phys. Rev. 63, 185 (1943).

[5] E. Feinberg, On the propagation of radio waves along an imperfect surface, J. Phys. (U.S.S.R.) 10, 410 (1946).

[6] K. Furutsu, Propagation of electromagnetic waves over a flat earth across a boundary separating different media and coastal refraction. J. Radio Research Labs. (Japan) $\boldsymbol{2}, 1$ (June 1955).

[7] J. R. Wait, Mixed path ground wave propagation: 1 Short distances, J. Research NBS 57, 1 (July 1956) RP2687; and J. R. Wait, J. Householder, 2. Larger distances, J. Research NBS 59, 19 (July 1957) RP2770.

[8] T. B. A. Senior, Radio propagation over a discontinuity in the earth's electrical properties-II. Coastal refraction, Proc. Inst. Elec. Engrs., Paper 201 R 104, Pt. C, 139 (March 1957).

Boulder Colo.

(Paper 64D1-38) 